\title{
ANALYSIS OF LARGE SCALE STRUCTURE USING PERCOLATION, GENUS AND SHAPE STATISTICS
}

\author{
VARUN SAHNI \\ Inter-University Centre for Astronomy \& Astrophysics, Post \\ Bag 4, Pune 411007, India
}

\begin{abstract}
.
We probe gravitational clustering in N-body simulations using geometrical descriptors sensitive to 'connectedness': the genus curve, percolation and shape statistics. As gravitational clustering advances, the density field in N-body simulations shows an increasingly pronounced departure from Gaussianity reflected in the changing shape of the percolation curve and the changing amplitude and shape of the genus curve. We feel that both genus and percolation curves provide complementary probes of large scale structure topology and could be used to discriminate between models of structure formation and the analysis of observational data such as galaxy catalogs and MBR maps. The filling factor in clusters \& superclusters at percolation is small indicating that matter is more likely to lie in filaments and pancakes. An analysis of 'shapes' in N-body simulations has shown that filaments are more pronounced than pancakes. To probe shapes of clusters and superclusters more rigorously we propose a new shape statistic which does not fit isodensity surfaces by ellipsoids (as done earlier). Instead our shape statistic is derived from fundamental properties of a compact body such as its volume $V$, surface area $S$, integrated mean curvature $C$, and connectivity (characterized by the Genus). The new shape statistic gives sensible results for topologically simple surfaces such as the ellipsoid, and for more complicated surfaces such as the torus.
\end{abstract}

\section{Introduction}

The Universe as we perceive it seems abundantly rich in visual form. Its large scale structure consisting of clusters and superclusters of galaxies has been variously perceived to be 'a cosmic web', 'network of surfaces', 
'sponge-like', bubble-like' etc. Attempts to describe its large scale features quantitatively have been made using a number of statistical indicators sensitive to the 'connectedness' of large scale structure including the genus curve and percolation statistics (Zeldovich 1982; Shandarin 1983; Gott, Melott, \& Dickinson 1986); minimal spanning trees (Barrow et al. 1995); Minkowski functionals; (Mecke et al. 1994) and statistics sensitive to shape (see Sahni \& Coles (1995) and references therein).

In this talk we assess the relative merits of genus and percolation curves by applying them to the same N-body simulations in an $\Omega=1$ Universe with scale-invariant initial conditions $P(k) \equiv\left\langle\left|\delta_{k}\right|^{2}\right\rangle \propto k^{n}, n=$ $-2,-1,0,+1$, (for simplicity we show results only for $n=-2$ which may be considered the lower limit of the slope of the initial spectrum on galaxy scales). Results are shown at several epochs each characterized by the scale of nonlinearity, $k_{N L}$, at that epoch measured in units of the fundamental mode $2 \pi / L$, where $L$ is the length of the simulation box. N-body simulations were performed on a $128^{3}$ grid using a particle-mesh algorithm (Melott $\&$ Shandarin 1993). A reduced grid of size $64^{3}$ was used to construct density fields from particle positions and the analysis of percolation and genus curves was then performed on these fields.

We also introduce a new statistic sensitive to shape based on Minkowski functionals.

\section{Growth of non-Gaussianity during Cosmological gravitational clustering.}

Conventional models of gravitational clustering usually assume that primordial density perturbations had a scale-invariant Harrison-Zeldovich spectrum and were distributed in the manner of a Gaussian random field. Arguments which support this hypothesis stem from the central limit theorem and the Inflationary paradigm. The non-Gaussianity which we observe in the Universe today (clusters, superclusters, voids) is attributed to nonlinear evolution and the resulting phase correlation between modes. Two robust and widely used statistical indicators of clustering are the probability density function (PDF) and the two point correlation function $\xi(r)$. However neither characterizes the nonlinear distribution of matter uniquely. The two point correlation function $\xi(x)=\int d^{3} \vec{k} \exp (i \vec{k} \cdot \vec{x}) P(k)$ being sensitive only to the power spectrum $P(k) \equiv\left\langle\left|\delta_{k}\right|^{2}\right\rangle$ and not to the phases $\phi_{k}$ of individual modes $\left[\delta(\vec{k})=\left|\delta_{k}\right| \exp \left(i \phi_{k}\right)\right]$ misses features arising because of phase correlations in the nonlinear regime. On the other hand, the PDF does not characterize a distribution uniquely in the nonlinear regime: distributions with identical PDF's can have very different topological properties and, conversely, distributions differing in their PDF's may have identical 
geometrical properties (this happens for instance in the case of distributions related by a mapping $\delta_{N L}=F\left(\delta_{L I N}\right)$, such as the log-normal).

\section{Percolation and Genus Curves}

It is clear that traditional indicators of clustering: the two point correlation function and the probability density function, must be complemented in the non-linear regime if we are to get a better understanding of the issue of nonGaussianity. One way of achieving this is to use geometrical measures which are sensitive to the connectedness of a distribution. Two such indicators percolation and the genus curve, will be studied in this section, a third shape statistics, will be discussed in the next.

One of the aims of percolation theory is to study the connectedness of structure as a function of the density threshold. Varying the density threshold from a high to low value, leads to a 'percolation transition' as the volume fraction in the largest cluster changes rapidly from almost zero to unity when the density threshold crosses a critical value. It is convenient to characterize percolation in terms of filling factor - henceforth $F F$ - the total volume in all clusters/voids above/below the density contrast threshold divided by the simulation volume ${ }^{1}$. Gaussian random fields percolate at the critical filling factor $F F_{C} \simeq 16 \%$ regardless of the spectrum. Density fields evolving under gravitational instability typically percolate at lower levels of $F F_{C}$ depending upon the initial spectrum and the extent of non-linear evolution (Yess \& Shandarin 1996). Similar conclusions are also reached in the case of point like distributions although the natural reference in this case is the Poisson distribution (Klypin \& Shandarin 1993).

Earlier work on gravitational clustering focussed on $F F_{C}$ as a diagnostic measure (Shandarin 1983; Dominik \& Shandarin 1992; Klypin \& Shandarin 1993). However, although useful in probing the extent of nonlinear evolution $F F_{C}$ does have certain drawbacks, for instance it is sensitive to resolution, number of particles and sample geometry (Dekel \& West 1985). A powerful new statistic without the above limitations is the percolation curve (PC). Consider the volume fraction $v_{\max }$ defined as the ratio of the volume in the largest cluster/void to the total volume in all clusters/voids lying above/below a density contrast threshold. The percolation curve describes the volume fraction $v_{\max }$ as a function of the density contrast threshold (or filling factor).

The percolation curve is plotted in Fig. 1 for evolved density fields from $\mathrm{N}$-body simulations with the power-law initial spectrum $n=-2$. Percolation curves for clusters (thick solid lines) and voids (thick dashed lines) are plotted separately. Vertical thin solid and thin dashed lines show the

\footnotetext{
${ }^{1} F F$ is the cumulative probability distribution function: $F F=P\left(\delta>\delta_{T}\right)$.
} 
percolation density threshold below which clusters and above which voids percolate. From Fig. 1 we find that at very high (low) thresholds the number of clusters (voids) is very small and $v_{\max }<<1$. As the threshold is gradually decreased (increased) the volume fraction in the largest cluster (void) increases as clusters (voids) begin to merge until the percolation transition when the largest 'supercluster' (supervoid) spans the entire simulation box. (Decreasing (increasing) the density threshold corresponds to increasing the filling factor for clusters (voids).) From Fig. 1 we see that as the simulation evolves $\delta_{C}$ increases monotonically as power in longer wavelengths causes structures to form and align on increasingly larger scales. For spectra with lesser long range power such as $n=0, \delta_{C}$ initially increases but later begins to decrease signaling the formation of small, isolated clumps (not shown) (Sahni, Sathyaprakash \& Shandarin 1997a).

An analysis similar to percolation can also be performed using the genus curve (GC) which can be formally expressed as an integral over the Gaussian curvature $K$ of the iso-density surfaces $S_{\nu}$ lying above/below a density threshold $\nu=\delta / \sigma_{\delta}$ by the Gauss-Bonnet theorem: $4 \pi G(\nu)=-\int_{S_{\nu}} K d A$. For Gaussian Random fields the genus curve has a 'bell shaped' form: $G(\nu)=A\left(1-\nu^{2}\right) \exp \left(-\nu^{2} / 2\right)$ (Hamilton et al. 1986; Gott et al. 1987; Gott et al. 1989). (An analytical expression for the genus in the weakly non-linear regime has been obtained in (Matsubara 1994).) Multiply connected surfaces have $G \geq 0$ while simply connected have $G<0$. The upper right hand panels of Fig. 1 show the genus curve plotted as a function of the density contrast. It is interesting to note that zero-crossings of the genus curve are quite close to the percolation threshold for both clusters and voids. This reflects the fact that the structure transforms from simply connected to multiply connected at the zero-crossing of $G$ which allows it to percolate. One can discern a strong increase in non-Gaussianity as the simulation evolves, reflected by an evolution in shape of both percolation and genus curves.

Instead of plotting GC and PC against the density contrast (which is not a normalized quantity), it may be more appropriate to plot them against the filling factor. This helps to distinguish between distributions related by a mapping $\delta \rightarrow f(\delta)$ (such as the log-normal) which have identical topological properties but can have quite different PDF's. The lower panels in fig. 1 show PC, GC for clusters (solid) and voids (dashed) plotted against FF. The three vertical lines show the filling factor at percolation for clusters, voids and Gaussian random fields with identical spectra. Both percolation and genus curves now resemble 'hysteresis' curves, the area between void and cluster curves indicating the degree of non-Gaussianity in the distribution. For PC we notice a marked increase in non-Gaussianity reflected in the increasing difference between percolation thresholds for clusters and 
voids measured by: $F F_{C}$ (voids) $-F F_{C}$ (clusters). The genus curve does not appear to evolve much, which is surprising. However the amplitude of GC does decrease with epoch, an effect which is more pronounced for spectra with greater small scale power, and which we attribute to the rapid build up of phase correlations due to nonlinear mode coupling during advanced gravitational clustering (Sahni, Sathyaprakash \& Shandarin 1997a).

Comparing the geometrical properties of a distribution to a featureless Gaussian, one can make statements regarding its 'connectedness or topology'. In Fig. 1 we have indicated the percolation threshold of Gaussian random fields by a dotted vertical line. Comparing these percolation thresholds with those of clusters and voids we conclude that percolation is 'easier' for clusters and more 'difficult' for voids. Clusters percolating at lower $F F$ than Gaussian are said to possess a 'network-like' topology. Voids on the other hand percolate at higher $F F$ than Gaussian and so have a 'bubble-like' topology. This appears to be a generic feature of most models of gravitational clustering with a reasonable amount of long-wavelength power in the initial spectrum (i.e $n \leq 0)$ (Sahni, Sathyaprakash \& Shandarin 1997a).

\section{Shape-statistics}

As discussed in the last section, gravitating systems clustering from Gaussian initial conditions percolate at low values of the filling factor. For CDM \& CHDM models the filling factor can be as small as $2 \%-7 \%$, much smaller than the $16 \%$ expected for a random Gaussian field (Klypin \& Shandarin 1993). This immediately suggests that the percolating phase is more likely to be sheet or filament-like since such distributions are likely to occupy a larger linear dimension (for an equal amount of mass) and will therefore percolate more easily. Some indication that this is indeed the case also comes from the Zeldovich approximation (Shandarin \& Zeldovich 1989).

A detailed study of 'shapes' in scale invariant models of gravitational clustering revealed that one dimensional 'filaments' are more abundant than two-dimensional 'sheets'. The filamentarity and pancakeness of structures grows with time, leading to the development of a long coherence length scale in simulations (Sathyaprakash, Sahni, \& Shandarin 1996). Exploring the 'connectedness' of large scale structure semi-analytically, Bond and collaborators recently concluded that clusters and superclusters appear to be interwoven in a 'cosmic web', with superclusters acting as cluster-cluster 'bridges'. More pronounced bridges are likely to form between clusters of galaxies which are aligned and close together (Bond, Kofman \& Pogosyan 1996).

The supercluster-void morphology is likely to vary for different scenario's of structure formation, it is unlikely that structure formation models 
based on gravitational instability will have identical topological properties as those based on string/texture models or explosions. We therefore feel that a study of shapes of superclusters/voids could help distinguish between different alternatives once galaxy catalogues become fully three dimensional and a detailed comparison between theory and observations becomes possible. Forthcoming redshift surveys such as SDSS and 2dF promise to shed more light on issues such as whether the 'great walls' appearing in Northern and Southern sky surveys are planar objects or are more like filaments or 'ribbons'.

Most shape statistics proposed so far study the shape of a collection of points by measuring its moment of inertia tensor, a procedure which is quite similar to fitting by an ellipsoid. Although this method has yielded some interesting results it is fair to say that none of the statistics applied to shapes is entirely satisfactory (Sathyaprakash, Sahni, Shandarin \& Fisher 1997). To illustrate this consider two examples: (1) the shape of an empty cup as determined from its moment of inertia tensor is approximately ellipsoidal whereas the cup is really a curved two-dimensional object. (The Zeldovich approximation in fact suggests that the caustic surfaces of the first pancake-like singularities are more likely to be curved than flat.) (2) A torus has both planar and filamentary properties; fitting with an ellipsoid would suggest an oblate shape, whereas a 'thin' torus is clearly more like a curved filament.

Results of N-body simulations clearly demonstrate that shapes of isodensity surfaces vary widely when viewed at different density thresholds. At high thresholds density peaks are mostly spheroidal, whereas at closer to percolation thresholds, surfaces get rather 'spongy' with a complicated topology.

To assess the shapes of objects which may be topologically non-trivial, we have recently proposed a shape statistic based on the four Minkowski functionals of a compact surface: (i) its Volume $V$, (ii) surface area $S$, (iii) integrated mean curvature: $C=\frac{1}{2} \int\left(\kappa_{1}+\kappa_{2}\right) d S$, and (iv) Genus $G$.

From $V, S, C$ and $G$ we construct three dimensionful and two dimensionless shape functions (Sahni, Sathyaprakash \& Shandarin 1997b). The dimensionful shape functions are: $\mathcal{H}_{1}=V / S, \mathcal{H}_{2}=S / C$ and $\mathcal{H}_{3}=C$. (For multiply connected surfaces $C / G$ may be more appropriate than $C$.) The pair of dimensionless shape functions $\mathcal{K} \equiv\left(\mathcal{K}_{1}, \mathcal{K}_{2}\right)$ is constructed from $\mathcal{H}_{i}$ : $\mathcal{K}_{1}=\frac{\mathcal{H}_{2}-\mathcal{H}_{1}}{\mathcal{H}_{2}+\mathcal{H}_{1}}, \mathcal{K}_{2}=\frac{\mathcal{H}_{3}-\mathcal{H}_{2}}{\mathcal{H}_{3}+\mathcal{H}_{2}},\left(\mathcal{K}_{1,2} \leq 1\right)$.

The shape functions are given below for certain 'idealized surfaces':

(1) pancake with vanishing thickness: $\mathcal{H}_{3} \simeq \mathcal{H}_{2}>>\mathcal{H}_{1}$ and $\mathcal{K} \simeq(1,0)$,

(2) filament with infinitesimal diameter: $\mathcal{H}_{3}>>\mathcal{H}_{2} \simeq \mathcal{H}_{1}$ and $\mathcal{K} \simeq$ $(0,1)$,

(3) sphere: $\mathcal{H}_{3} \simeq \mathcal{H}_{2} \simeq \mathcal{H}_{1}$ and $\mathcal{K} \simeq(0,0)$, 
(4) ribbon: $\mathcal{H}_{3}>>\mathcal{H}_{2}>>\mathcal{H}_{1}$ and $\mathcal{K} \simeq(1,1)$.

Realistic surfaces will be represented as points on a 'shape plane' $\left(\mathcal{K}_{1}, \mathcal{K}_{2}\right)$, with ideal pancakes, filaments, ribbons and spheres defining its four vertices: $(1,0),(0,1),(1,1),(0,0)$.

To demonstrate the effectiveness of the shape statistic we apply it to two surfaces- an ellipsoid and a torus. The surface of the triaxial ellipsoid has the parametric form

$$
\mathbf{r}=a(\sin \theta \cos \phi) \hat{x}+b(\sin \theta \sin \phi) \hat{y}+c(\cos \theta) \hat{z}
$$

where $0 \leq \phi \leq 2 \pi, 0 \leq \theta \leq \pi$.

In table 1 we show results for deformations of this ellipsoid.

TABLE 1. Deformations of a triaxial ellipsoid with axis $a, b, c$. The shape functions $V / S, S / C$, $C$ have dimensions of length, $\left(\mathcal{K}_{1}, \mathcal{K}_{2}\right)$ are dimensionless.

\begin{tabular}{cccccc}
\hline$a, b, c$ & deformation & $V / S$ & $S / C$ & $C$ & $\left(\mathcal{K}_{1}, \mathcal{K}_{2}\right)$ \\
\hline$(100,100,100)$ & sphere $\rightarrow$ filament & 100.00 & 100.00 & 100.00 & $(0,0)$ \\
$(100,80,80)$ & & 85.45 & 86.12 & 86.97 & $\left(3.9 \times 10^{-3}, 4.9 \times 10^{-z}\right.$ \\
$(100,50,50)$ & & 58.51 & 61.92 & 69.01 & $\left(2.8 \times 10^{-2}, 5.4 \times 10^{-z}\right.$ \\
$(100,20,20)$ & & 25.04 & 29.22 & 54.68 & $\left(7.7 \times 10^{-2}, 0.30\right)$ \\
$(100,10,10)$ & & 12.67 & 15.32 & 51.50 & $\left(9.5 \times 10^{-2}, 0.54\right)$ \\
$(100,3,3)$ & & 3.82 & 4.70 & 50.19 & $(0.10,0.83)$ \\
\hline$(100,100,100)$ & sphere $\rightarrow$ pancake & 100.00 & 100.00 & 100.00 & $(0,0)$ \\
$(100,100,80)$ & & 91.99 & 92.89 & 93.63 & $\left(4.9 \times 10^{-3}, 3.9 \times 10^{-3}\right.$ \\
$(100,100,50)$ & & 72.45 & 80.75 & 85.46 & $\left(5.4 \times 10^{-2}, 2.8 \times 10^{-2}\right.$ \\
$(100,100,20)$ & & 36.58 & 68.45 & 79.88 & $\left(0.30,7.7 \times 10^{-2}\right)$ \\
$(100,100,10)$ & & 19.42 & 65.28 & 78.90 & $\left(0.54,9.5 \times 10^{-2}\right)$ \\
$(100,100,3)$ & & 5.98 & 63.88 & 78.57 & $(0.83,0.10)$ \\
\hline$(100,100,3)$ & pancake $\rightarrow$ filament & 5.98 & 63.88 & 78.57 & $(0.83,0.10)$ \\
$(100,70,3)$ & & 5.97 & 52.27 & 67.32 & $(0.80,0.13)$ \\
$(100,30,3)$ & & 5.90 & 27.81 & 54.88 & $(0.65,0.33)$ \\
$(100,10,3)$ & & 5.47 & 10.79 & 50.88 & $(0.33,0.65)$ \\
$(100,3,3)$ & & 3.82 & 4.70 & 50.19 & $(0.10,0.83)$ \\
\hline
\end{tabular}

Next consider a topologically more complicated surface, the elliptical torus

$$
\mathbf{r}=(b+c \sin \phi) \cos \theta \hat{x}+(b+c \sin \phi) \sin \theta \hat{y}+a(\cos \phi) \hat{z}
$$

where $a, c<b, 0 \leq \theta, \phi<2 \pi$. Table 2 shows shape functions for deformations of an elliptical torus which are illustrated in Fig. 2. (The inner tube of the torus has an elliptical cross-section with $a \& c$ being respectively, radii of curvature perpendicular and parallel to the plane of the torus; $a=c$ gives the usual circular torus.) 
TABLE 2. Shape functions for an elliptical torus with axis $b, a, c,(b>a, c)$.

\begin{tabular}{cccccc}
\hline$b, a, c$ & Morphology & $\left(\mathcal{K}_{1}, \mathcal{K}_{2}\right)$ & $V / S$ & $S / C$ & $C$ \\
\hline$(100,99,3)$ & Pancake1 & $\left(0.90,2.9 \times 10^{-2}\right)$ & 7.05 & 136.89 & 144.94 \\
$(100,3,99)$ & Pancake2 & $(0.88,0.20)$ & 7.05 & 114.66 & 173.03 \\
$(100,3,3)$ & Filament & $(0.14,0.93)$ & 4.5 & 6.0 & 157.08 \\
$(150,20,2)$ & Ribbon1 & $(0.70,0.80)$ & 4.64 & 25.88 & 235.56 \\
$(150,2,20)$ & Ribbon2 & $(0.70,0.80)$ & 4.64 & 25.87 & 235.65 \\
$(20,19,19)$ & Sphere-with-hole & $(0.14,-0.09)$ & 28.5 & 38.0 & 31.42 \\
\hline
\end{tabular}

We find that qualitatively similar deformations of the ellipsoid and torus give rise to similar values of the shape statistic which demonstrates that the statistic is robust.

The shape statistic we propose has several advantages over earlier shapefinders which were insensitive to topology (Sahni, Sathyaprakash \& Shandarin 1997b; Sathyaprakash, Sahni, Shandarin \& Fisher 1997). As a result it can successfully be applied to superclusters and voids in N-body simulations and in galaxy catalogues even when these occur at relatively low density threshold and are therefore not necessarily simply connected. ${ }^{2}$

\section{Conclusions.}

Non-Gaussianity in simulations of large scale structure and in galaxy catalogs can be studied using geometrical descriptors such as percolation, genus curves and shape statistics. Geometrical indicators are sensitive to the 'connectedness of a distribution and complement the two-point correlation function and the PDF. As gravitational clustering advances, the density field in N-body simulations shows an increasingly pronounced departure from Gaussianity reflected in the changing shape of the percolation curve and the changing amplitude and shape of the genus curve, we conclude that both genus and percolation curves provide complementary probes of large scale structure topology and can be used to discriminate between models of structure formation and the analysis of observational data such as galaxy catalogs and MBR maps. The smallness of the filling factor in clusters \& superclusters at percolation indicates that a bulk of the matter is likely to lie in filaments and pancakes. An analysis of 'shapes' in N-body simulations shows that filaments grow more pronounced as the simulation evolves and are more prominent for spectra with greater large scale power.

\footnotetext{
${ }^{2}$ The number of clusters peaks at thresholds just below $F F_{C}$ making this a useful threshold at which to determine cluster shapes using our statistic. (At $F F>F F_{C}$ most clusters link up to form a percolating supercluster whereas at $F F<<F F_{C}$ only a few clusters are present and these gradually disappear as $F F \rightarrow 0$.)
} 
To probe 'shapes' more rigorously we introduce a new shape-statistic which studies shapes of compact surfaces (iso-density surfaces in galaxy surveys or N-body simulations) without fitting them to ellipsoidal configurations as done earlier. The new shape-indicators arise from simple, geometrical considerations and are derived from fundamental properties of a compact body such as its volume $V$, surface area $S$, integrated mean curvature $C$, and Genus. The new shape statistics can be applied to topologically simple and complicated surfaces and appears to be quite robust.

Acknowledgments: The work reported here was done in collaboration with B. S. Sathyaprakash and Sergei Shandarin to whom I express my gratitude and thanks for many years of fruitful interaction. I also acknowledge stimulating discussions with Sanjeev Dhurandhar and Somak Raychaudhury.

\section{References}

Barrow, J.D., Bhavsar, S.P. \& Sonoda, D.H. 1985 MNRAS, 21617.

Bond, J.R., Kofman, L. \& Pogosyan, D. 1996, Nature, 380, 603.

de Lapparent, V., Geller, M.J. \& Huchra, J.P. 1991, ApJ, 369, 273

Dekel A. \& West M.J. 1985, ApJ, 288, 411.

Dominik K. \& Shandarin S.F. 1992, ApJ, 393, 450

Gott, J.R., Melott, A.L. \& Dickinson, M. 1986, ApJ, 306, 341

Gott, J.R., Weinberg, D.H. \& Melott, A.L., 1987, ApJ, 319, 1.

Gott, J.R. et al. 1989, ApJ, 340, 625.

Hamilton, A.J.S., Gott, J.R. \& Weinberg, D.H., 1986, ApJ, 309, 1.

Klypin A.A. \& Shandarin S.F. 1993, ApJ, 413, 48

Matsubara, T. 1994, ApJ, 434, L43

Melott, A.L. \& Shandarin, S.F. 1993, ApJ, 410, 469

Mecke, K.R, Buchert, T. \& Wagner, H., 1994, Astron. Astrophys., 288, 697.

Melott, A.L. 1990, Physics Reports, 193, 1

Sahni V. \& Coles P., 1995, Physics Reports, 262, 1

Sahni V., Sathyaprakash B.S. \& Shandarin S.F. 1997a, ApJ 476, L1-L5.

Sahni V., Sathyaprakash B.S. \& Shandarin, S.F. 1997b, ApJ, submitted.

Sathyaprakash B.S., Sahni V. \& Shandarin S.F. 1996, ApJ, 462, L5-L8.

Sathyaprakash B.S., Sahni V., Shandarin S.F. \& Fisher K. 1997, MNRAS, submitted Shandarin, S.F. 1983, Soviet Astron. Lett., 9, 104

Shandarin, S.F. \& Zeldovich Ya. B. 1989, Rev. Mod. Phys., 61, 185

Yess C. \& Shandarin S.F. 1996, ApJ, 465, 2

Zeldovich Ya. B. 1982, Soviet Astron. Lett., 8, 102 

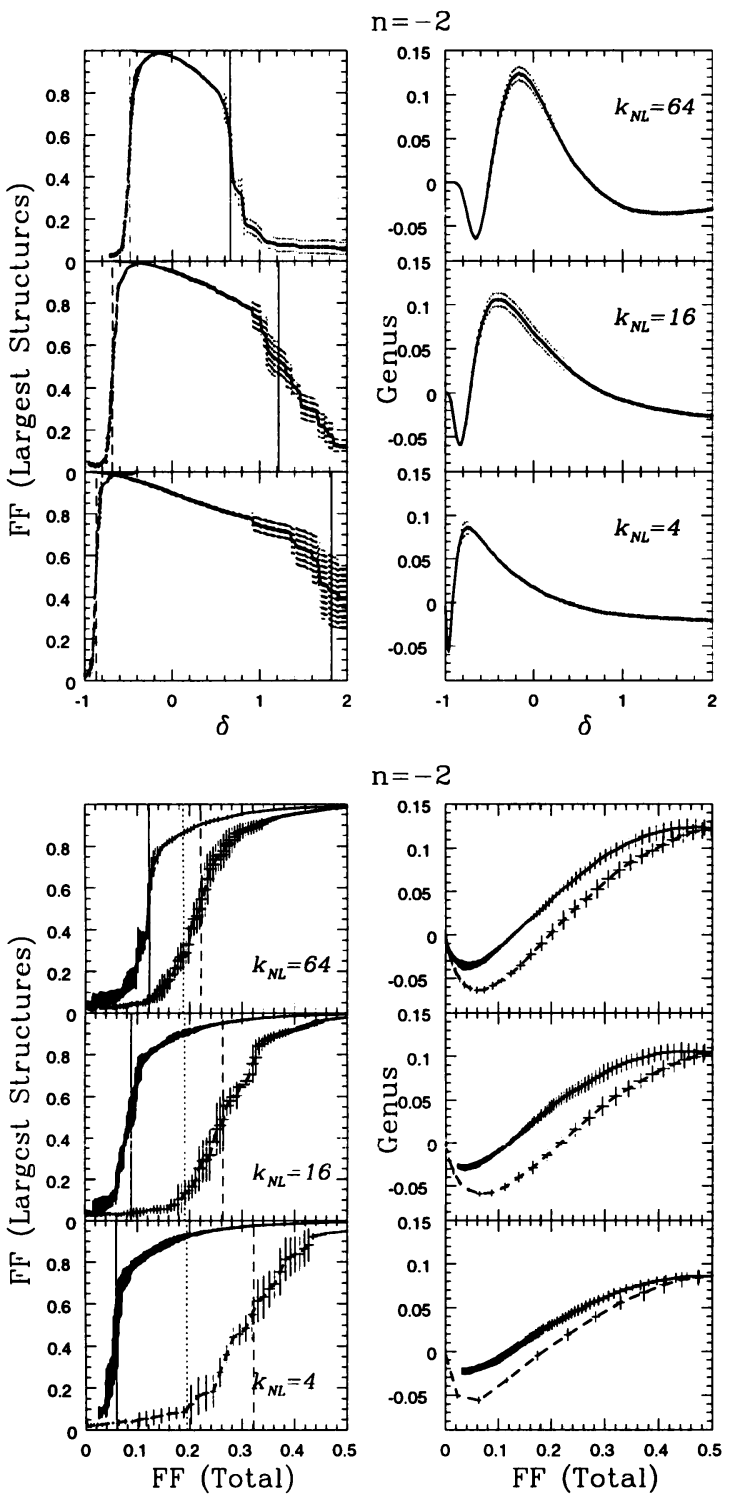

Figure 1. Percolation (left panels) and genus (right panels) curves are shown as functions of the density contrast $\delta$ (above) and filling factor (below) for a scale free initial spectrum $n=-2$. In plots showing PC (left panels), solid (dashed) curves correspond to the volume fraction in the largest cluster (void) $-v_{\max }$. Vertical solid (dashed) lines show the threshold describing percolation between opposite faces of the cube for clusters (voids). The thin dotted line in the lower left hand panel shows the filling factor at percolation for a Gaussian random field with the same power spectrum as of evolved density fields (for details see Sahni, Sathyaprakash \& Shandarin 1997a). 
Pancake1

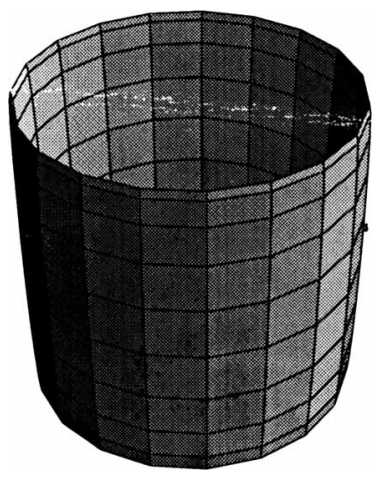

Ribbon1

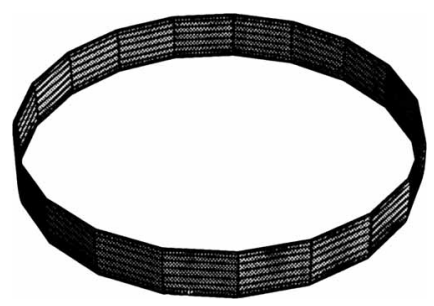

Filament

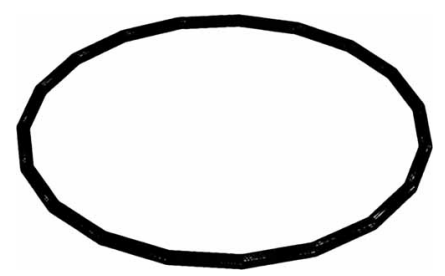

Pancake2

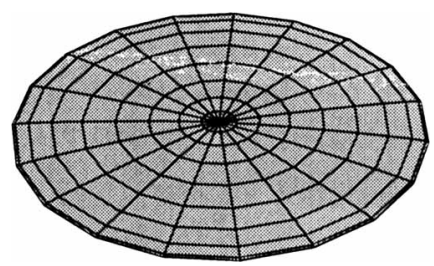

Ribbon2

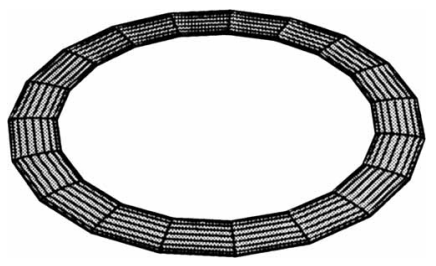

Sphere-with-hole

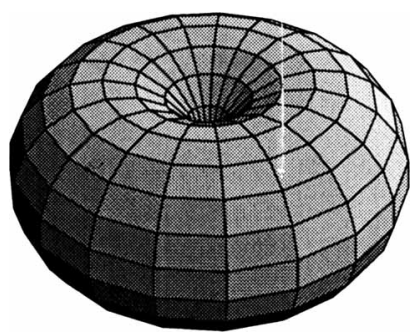

Figure 2. Deformations of an elliptical torus. 